\title{
Mobile Introns Shape the Genetic Diversity of Their Host Genes
}

\author{
Jelena Repar and Tobias Warnecke ${ }^{1}$ \\ Molecular Systems Group, MRC London Institute of Medical Sciences (LMS), and Institute of Clinical Sciences, Faculty of Medicine, \\ Imperial College London, W12 0NN, United Kingdom \\ ORCID ID: 0000-0002-4936-5428 (T.W.)
}

\begin{abstract}
Self-splicing introns populate several highly conserved protein-coding genes in fungal and plant mitochondria. In fungi, many of these introns have retained their ability to spread to intron-free target sites, often assisted by intron-encoded endonucleases that initiate the homing process. Here, leveraging population genomic data from Saccharomyces cerevisiae, Schizosaccharomyces pombe, and Lachancea kluyveri, we expose nonrandom patterns of genetic diversity in exons that border self-splicing introns. In particular, we show that, in all three species, the density of single nucleotide polymorphisms increases as one approaches a mobile intron. Through multiple lines of evidence, we rule out relaxed purifying selection as the cause of uneven nucleotide diversity. Instead, our findings implicate intron mobility as a direct driver of host gene diversity. We discuss two mechanistic scenarios that are consistent with the data: either endonuclease activity and subsequent error-prone repair have left a mutational footprint on the insertion environment of mobile introns or nonrandom patterns of genetic diversity are caused by exonic coconversion, which occurs when introns spread to empty target sites via homologous recombination. Importantly, however, we show that exonic coconversion can only explain diversity gradients near intron-exon boundaries if the conversion template comes from outside the population. In other words, there must be pervasive and ongoing horizontal gene transfer of self-splicing introns into extant fungal populations.
\end{abstract}

KEYWORDS genetic diversity; self-splicing introns; mutagenic; gene conversion; homing endonuclease

ELF-splicing introns are selfish elements with a broad but patchy phylogenetic distribution. Found in transfer RNA (tRNA), ribosomal RNA (rRNA) and (occasionally) proteincoding genes in bacteria and archaea, they are particularly numerous in mitochondrial genomes of fungi and plants where they have invaded genes encoding components of the electron transport chain (ETC) (Lambowitz and Belfort 1993). In many instances, fungal self-splicing introns have remained mobile, as demonstrated by experiments that track invasion capacity by crossing intron-containing with intron-

Copyright @ 2017 Repar and Warnecke

doi: https://doi.org/10.1534/genetics.116.199059

Manuscript received December 8, 2016; accepted for publication February 7, 2017; published Early Online February 13, 2017.

Available freely online through the author-supported open access option.

This is an open-access article distributed under the terms of the Creative Commons Attribution 4.0 International License (http://creativecommons.org/licenses/by/4.0/, which permits unrestricted use, distribution, and reproduction in any medium, provided the original work is properly cited.

Supplemental material is available online at http://www.genetics.org/content/early/ 2017/02/13/genetics.116.199059.supplemental.

${ }^{1}$ Corresponding author: MRC London Institute of Medical Sciences (LMS), Hammersmith Hospital Campus, Du Cane Rd., London W12 ONN, United Kingdom. E-mail: tobias. warnecke@imperial.ac.uk free yeast strains (Jacquier and Dujon 1985; Wenzlau et al. 1989; Lazowska et al. 1994; Paschke et al. 1994) and intron presence/absence polymorphisms across natural populations of Saccharomyces cerevisiae (Wolters et al. 2015), Schizosaccharomyces pombe (Zimmer et al. 1987), and Lachancea kluyveri (Jung et al. 2012). For the majority of self-splicing introns in $S$. cerevisiae, spreading to an intron-free location is initiated by a homing endonuclease that is encoded in the intron itself and binds a large ( 20-30 bp), often singular target motif with high affinity (Jacquier and Dujon 1985; Moran et al. 1992). Following cleavage of the intron-free homing site, the intron-containing copy of the mitochondrial genome is used as a template for homologous recombination (HR), resulting in the conversion of an intron-free to an introncontaining locus. Once gained, introns can be lost again either through fortuitous deletion or through a gene conversion event that involves an intronless complementary DNA (cDNA) produced by reverse transcriptase (RT) activity (Levra-Juillet et al. 1989), as has been proposed for spliceosomal introns (Cohen et al. 2012). In the absence of selection for intron retention, cycles of intron gain and loss ensue 
(Goddard and Burt 1999), accompanied by recurrent endonuclease activity that predictably targets the very same recognition site.

Here, prompted by reports of possible mutational hotspots in the vicinity of self-splicing intron (Hensgens et al. 1983; Zimmer et al. 1987; Foury et al. 1998), we consider what impact these invasion-loss cycles have on the genetic diversity of the host gene. In particular, we consider the possibility that endonuclease-mediated cleavage and subsequent repair might be mutagenic. Although HR is generally considered to be high fidelity, it can carry nonnegligible mutagenic risks, depending on the precise nature of the repair process and whether error-prone polymerases are involved in DNA resynthesis (Rodgers and McVey 2016). Pertinently, Hicks et al. (2010) observed increased mutation rates during doublestrand break (DSB) repair at the mating type (MAT) locus of $S$. cerevisiae, which is cleaved by the endonuclease $\mathrm{HO}$ and subsequently repaired via HR (Hicks et al. 2010).

Mutagenic side effects associated with endonuclease activity have also come into sharp focus recently with the widespread adoption of targetable endonucleases for genome engineering. The principal concern here has been to identify and reduce off-target activity (Cho et al. 2014; Kleinstiver et al. 2016). However, endonuclease activity can also have undesired on-target effects. Notably, nonhomologous end joining (NHEJ) downstream of Cas9-mediated cleavage is associated with an increased risk of indel formation (van Overbeek et al. 2016). This has prompted the development of Cas9 derivatives that nick rather than cleave DNA (Cong et al. 2013; Mali et al. 2013), shifting repair pathway choice away from NHEJ and toward HR.

We reasoned that one way to develop a greater understanding of such on-target mutagenicity would be to study endonucleases in their native genomic context. If endonuclease activity is indeed mutagenic, cleavage and repair might have left a detectable imprint on population-wide genetic variation around the cleavage site. In search of such an imprint, we survey recent high-quality population genomic data from S. cerevisiae, S. pombe, and L. kluyveri to characterize single nucleotide polymorphism (SNP) patterns in exons flanking mitochondrial self-splicing introns.

\section{Materials and Methods}

\section{Data acquisition and identification of polymorphic sites}

We obtained the sequences of $93 \mathrm{~S}$. cerevisiae mitochondrial genomes, originating from a recent high-coverage resequencing effort (Strope et al. 2015), from John Wolters (Binghamton University). Baiting BLAST searches (blastn, $E$ value $<1 E-9$ ) with the terminal exons of $c o b$ and cox 1 from the reference S288C genome, we identified unique full-length $\operatorname{cob}$ and $\operatorname{cox} 1$ genes in all strains, capturing both coding exons and intervening introns. We aligned the 94 sequences (93 plus the S288C reference genome) for each gene together with $50 \mathrm{nt}$ of upstream/ downstream flanking DNA using MUSCLE v. 3.8.31 (Edgar
2004) and manually surveyed the alignment around intronexon boundaries for alignment errors or conspicuous outliers. As a consequence of this manual inspection, we conservatively excluded strain YJM1250, which exhibits an unusual multinucleotide difference at the $5^{\prime}$ end of exon 6 , which would have further exacerbated the SNP density gradient reported below. We also excluded strain YJM1399, whose mitochondrial genome was previously found to be more closely related to S. paradoxus (Wolters et al. 2015). Polymorphic sites in $c o b$ and cox 1 were therefore identified from the alignment of 92 sequences. In inferring distances to the nearest intron-exon boundary, we only considered introns present in the reference genome. This is conservative since residues that are inferred to be exon internal might in fact be close to an intron present in the population but not the reference genome, thus overestimating mutations internal to the exon.

For the analysis of the MAT locus and nuclear spliceosomal introns, chromosomes for the 91 resequenced yeast genomes and S228C were downloaded via Batch Entrez based on their GenBank accessions in Strope et al. (2015) and the Saccharomyces Genome Database (http://www.yeastgenome.org), respectively. Identification and alignment of the MAT $\alpha$ (which is reported in all genome assemblies in favor of MATa) and Z1 regions based on the S288C annotation were straightforward, given the exceptional conservation levels reported below. Genes containing nuclear spliceosomal introns in S228C were identified based on GenBank annotations. The terminal exons of these genes (required minimum length $>1 \mathrm{nt}$ ) were then blasted against the remaining genomes (blastn, $E$ value $<1 E-9$ ). Homologous sequences were extracted for cases where both terminal exons were at least $70 \mathrm{nt}$ long, identified as the only hits in the BLAST query, located on the same chromosome and strand, and $<3 \mathrm{~kb}$ apart (covering the empirical intron-containing gene length distribution in S. cerevisiae). Homologous sequences recovered in at least 80 strains were then aligned using MUSCLE with default parameters.

We obtained cox 1 and cob coding sequences for $18 \mathrm{~L}$. kluyveri strains from Paul Jung (University of Luxembourg). Intron positions in these strains were taken from supplemental material, figure S2 of Jung et al. (2012). Following alignment of these sequences (using the same parameters employed for S. cerevisiae) and subsequent manual inspection, we conservatively excluded strains CBS10367 and CBS10368 because of conspicuous divergence at the $5^{\prime}$ end of exon 4 .

In inferring distances to the nearest intron-exon boundary, we considered all intron insertion sites observed across the 18 L. kluyveri strains by Jung et al. (2012).

We obtained variant calls across $161 \mathrm{~S}$. pombe strains (Jeffares et al. 2015) from Daniel Jeffares (available at https://danieljeffares.com/data/). In the absence of highquality de novo mitochondrial assemblies for these strains and unknown intron presence/absence variability, we only considered introns present in the $S$. pombe reference genome when inferring distances to the nearest intron-exon boundary. 
Variant calls across 1135 Arabidopsis thaliana chloroplast and mitochondrial genomes were obtained from the $1001 \mathrm{Ge}$ nomes Project (Cao et al. 2011) (via A. Farlow). In the absence of de novo mitochondrial/chloroplast assemblies for these strains, we only considered introns present in the A. thaliana TAIR10 reference genome when inferring distances to the nearest intron-exon boundary.

Human cob and cox1 coding sequences and associated polymorphism data were obtained from the Mitomap database (Lott et al. 2013). Mock intron-exon boundaries in the human sequences were placed at orthologous positions as identified from human-S. cerevisiae-L. kluyveri alignments for $\operatorname{cox} 1$ and $c o b$.

\section{Calculation of SNP densities and assessment of overlap with functional motifs}

Correlations were calculated across a 70-nt window from the boundary, which captures the regions of elevated and plateauing SNP density in all species. Further extending this region is not beneficial since increasingly fewer exons contribute to specific positions. For all species, SNP density at a given distance from the boundary is calculated across all pertinent exons as the number of polymorphic sites divided by the number of exons that contribute a site at that distance. Exons $<70 \mathrm{nt}$ will therefore not make a contribution at all distances and the denominator for each distance is adjusted accordingly. Note that any one site will only be counted once, at the distance to the nearest intron, rather than being counted twice, i.e., in relation to the upstream and downstream intron.

We use structural information provided by the Group I Intron Sequence and Structure Database (GISSD) (Zhou et al. 2007) and the Zimmerly lab (http://www.fp.ucalgary.ca/ group2introns/) to assess the degree to which polymorphic exonic sites were involved in pairing to intronic residues as part of the self-splicing process. Overlap with endonuclease cleavage sites was assessed based on cleavage motifs defined in REBASE v608 (Roberts et al. 2010). Experimentally mobile $S$. cerevisiae introns were defined as in Lambowitz and Belfort (1993). Polymorphisms were classified into synonymous and nonsynonymous according to the yeast mitochondrial code [National Center for Biotechnology Information (NCBI) transl_table $=3$ ] for $S$. cerevisiae and L. kluyveri and the standard code (NCBI transl_table $=1$ ) for $S$. pombe.

\section{Data availability}

Our analyses are fully based on publicly available data. Publications, websites, and databases from which the data were obtained are indicated throughout Materials and Methods.

\section{Results and Discussion}

\section{Elevated polymorphism density at the exonic boundaries of mitochondrial introns}

The $S$. cerevisiae mitochondrial reference genome harbors a single group I intron in the $21 \mathrm{~S}$ rRNA gene and multiple group I and II introns in the protein-coding genes $c o b$ and cox1 (Figure 1A). Whereas strong constraints on RNA structure and base pairing govern the evolution of tRNA and rRNA genes throughout most of their sequence, protein-coding genes contain synonymous sites that might in principle allow for a better mutational readout, particularly at short evolutionary time scales. We therefore focused our analysis on protein-coding genes. Using high-coverage genome assemblies of 92 S. cerevisiae strains (see Materials and Methods), we first considered SNP density as a function of distance from the nearest intron-exon boundary across cox 1 and cob exons. SNP density here is defined as the number of SNPs at a given distance ( $\leq 70 \mathrm{nt}$ ) from the nearest intron boundary, divided by the number of exons that contribute a nucleotide at that distance (i.e., taking into account that an exon of size $30 \mathrm{nt}$, for example, would not contribute to the denominator at distances >30 nt; see Materials and Methods for further details). We observe a marked increase in exonic SNP density as one approaches the intron-exon boundary (Kendall's $\tau=-0.23, P=0.01$, Figure $1 \mathrm{~B}$ ), consistent with previous reports of polymorphism clusters located at the exonic border of specific endonuclease-encoding introns (Hensgens et al. 1983; Foury et al. 1998). The 5' end of $\operatorname{cox} 1$ exon 6, previously proposed as a mutational hotspot (Foury et al. 1998), contributes to but does not chiefly drive this trend $(\tau=-0.17$, $P=0.05$ when cox 1 exon 6 is excluded).

In addition, the SNP density gradient is evident for both group I introns $(\tau=-0.19, P=0.04)$ and group II introns $\left(\tau=-0.24, P=0.01\right.$ ) and is broadly similar for $5^{\prime}$ and $3^{\prime}$ exon ends, with a marginally smaller contribution of $3^{\prime}$ exon ends (Supplemental Material, Figure S1). This might be linked to a greater fraction of nucleotides at $3^{\prime}$ exon termini being under selection to maintain splice-relevant base-pairing interactions with the neighboring intron (see below). Inevitably given the area of SNP enrichment, a substantial proportion of boundary-proximal polymorphisms are located in known endonuclease cleavage motifs $(17 / 24=71 \%$ of mutations within $20 \mathrm{nt}$ of the intron-exon boundary overlap homing endonuclease recognition sites; see Materials and Methods). This might be considered surprising. However, systematic mutagenesis experiments previously demonstrated that many single-nucleotide changes do not perturb target recognition and cleavage (Sargueil et al. 1990; Wernette et al. 1992). Indeed, the three SNPs recovered here that overlap a previously mutagenized endonuclease cleavage site (split across exons 4 and 5 of cox 1 ) had all been tested individually and found to have wild-type cutting efficacies (Sargueil et al. 1990).

\section{SNP density gradients are specifically associated with mobile introns}

Previous studies comparing pairs of strains (one with and one without a focal intron) had postulated the presence of mutational hotspots near intron-exon boundaries but lacked quantitative support (Hensgens et al. 1983; Zimmer et al. 1987; Foury et al. 1998). These studies had also speculated 


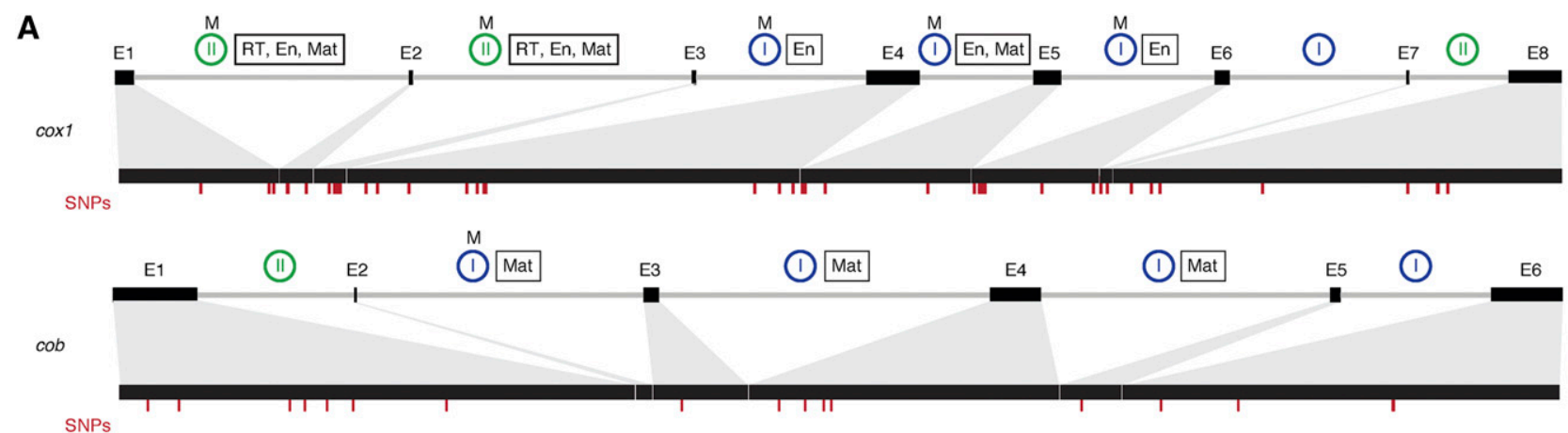

B

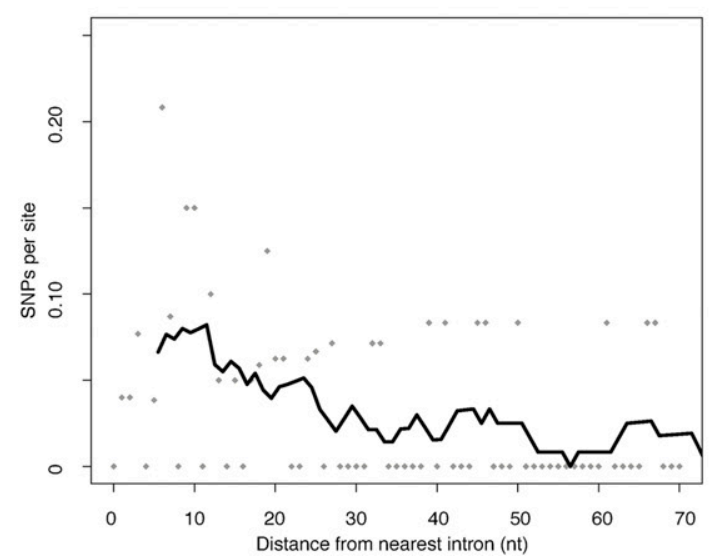

C

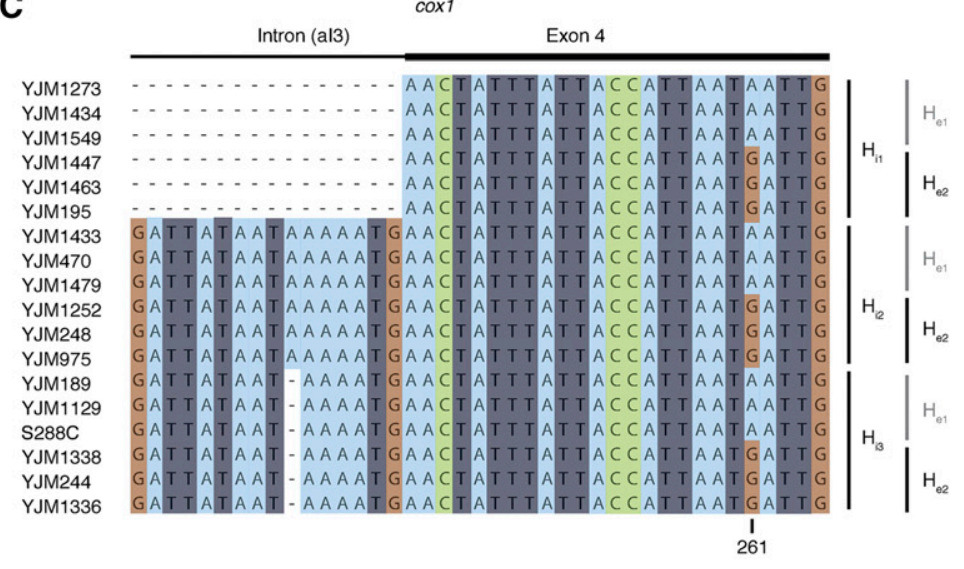

Figure 1 Elevated SNP density at mitochondrial intron-exon boundaries in S. cerevisiae. (A) Exon/intron structures of cox1 and cob, with exons depicted as black boxes connected by gray lines (introns). Introns known to be mobile (see Materials and Methods) are labeled (M) above blue or green circles that indicate group I and II introns, respectively. Introns labeled with a rectangle harbor ORFs that encode proteins with endonuclease (En), maturase (Mat), and/or RT activity. The locations of SNP are marked by red dashes. (B) SNP density as a function of distance from the nearest intron-exon boundary. SNPs per site (calculated across all cox1/cob exons as described in the main text) are indicated by gray dots. To illustrate the general trend, we also provide a density curve, derived by smoothing across 10-nt windows, moving in 1-nt steps. The curve starts at the center point of the first window rather than at 0 . (C) Excerpt from the cox1 alignment of $92 \mathrm{~S}$. cerevisiae strains, highlighting a short region at the junction of intron 3 and exon 4 across 18 strains. Three different intronic $\left(\mathrm{H}_{\mathrm{i1}-3}\right)$ and two different exonic $\left(\mathrm{H}_{\mathrm{e} 1-2}\right)$ haplotypes are evident, with all six possible combinations present in the population.

that mobility might be a causal factor in elevated nucleotide diversity, or more specifically, that variation was being introduced upon intron gain (Zimmer et al. 1987) or loss (Hensgens et al. 1983). The more extensive sampling of population genetic variation carried out here reveals that there is no perfect correspondence between polymorphisms and intron presence or absence (an illustrative example is shown Figure 1C), precluding straightforward attribution of novel exonic variation to intron gain or loss. However, we find strong support that mobility in general is key. Although an elevated SNP density is detectable when considering polymorphisms across all cob/cox1 exon termini, this effect is specifically driven by exon ends that adjoin mobile introns ( $\tau=-0.31, P=0.0007$, Figure 2A; mobility as defined by previous experimental research; see Materials and Methods). Exon ends bordering immobile mitochondrial introns do not show a similar enrichment for SNPs $(\tau=-0.04, P=0.64)$. Similarly, we find no SNP enrichment in the exonic borders of spliceosomal nuclear introns, which lack the capacity to excise themselves from their host messenger RNA (mRNA) and do not encode endonucleases or other mobility factors
( $\tau=0.08, P=0.37$, Figure 2B). In short, elevated SNP densities at intron-exon boundaries are confined to introns that are both self-splicing and mobile. Further support for a critical role of mobility comes from population genomic analysis of 1135 A. thaliana accessions (see Materials and Methods), whose mitochondrial and chloroplast genomes also harbor self-splicing introns embedded in protein-coding genes. However, unlike their fungal counterparts, these introns lack open reading frames that encode functional endonuclease, RT, or other domains that might mediate mobility and, like those of other land plants, are not mobile as a consequence (Bonen 2008). As predicted under a model where mobility is critically linked to elevated nucleotide diversity, we find no evidence for higher SNP densities near intron-exon boundaries in $A$. thaliana (combined $\tau=-0.04, P=0.65$, Figure 2C), albeit on a background of globally low mutation rates.

In $S$. cerevisiae, most mobile introns are located in cox1, with only a single intron in $c o b$ reported to be mobile in crossing experiments (Lambowitz and Belfort 1993) (Figure 1A). As a consequence, cox 1 exons exhibit SNP enrichment near the intron-exon boundary $(\tau=-0.34, P=0.0002)$, 
B $\quad$ C

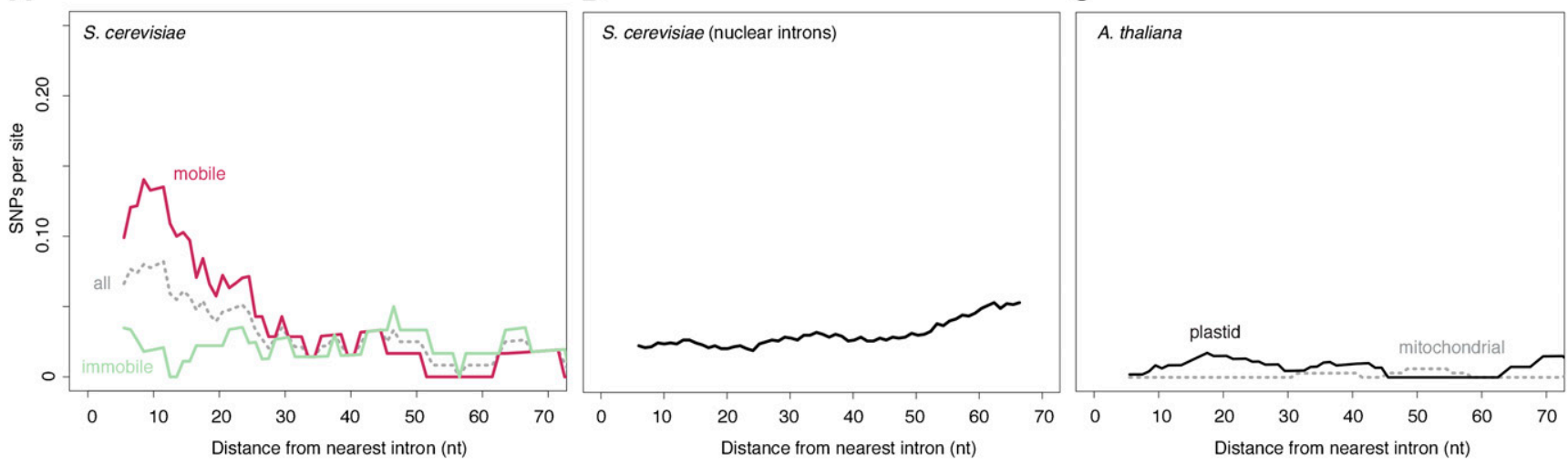

D
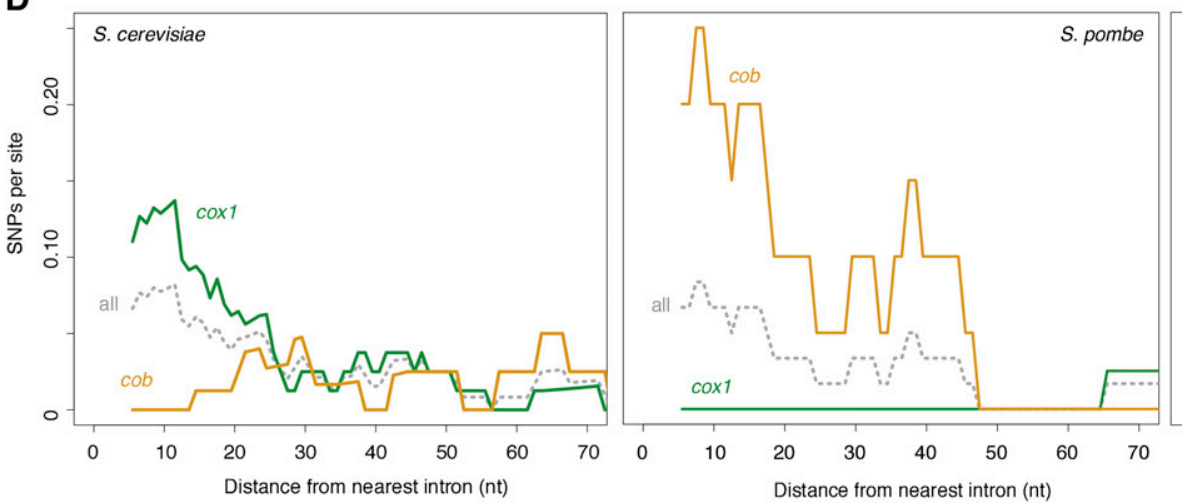

L. kluyveri

Figure 2 Elevated SNP density at intron-exon boundaries in different genes and species. (A) SNP density as a function of distance from the nearest intron-exon boundary across $S$. cerevisiae cox $1 / \mathrm{cob}$ exons experimentally determined to be mobile (see Materials and Methods) and the immobile remainder. The gray dotted line (all) indicates the combined mobile/immobile data and corresponds to the data shown in Figure 1B. (B) SNP density as a function of distance from the nearest intron-exon boundary across S. cerevisiae exons bordering spliceosomal nuclear introns. (C) SNP density as a function of distance from the nearest intron-exon boundary for introns in intron-containing mitochondrial (gray) or chloroplast (black) protein-coding genes of A. thaliana. (D) SNP density as a function of distance from the nearest intron-exon boundary for introns in the cob (yellow) and cox1 (green) genes of S. cerevisiae, S. pombe, and L. kluyveri. The gray dotted lines (all) indicate combined cox 1/cob data. For all panels, the trend lines are smoothed density curves as described in Figure 1B. In-text correlation coefficients are calculated from the underlying raw data.

whereas cob exons do not ( $\tau=0.07, P=0.48$ ). To rule out gene-specific factors rather than mobility in the genesis of nucleotide diversity, we examined mitochondrial proteincoding genes from $161 \mathrm{~S}$. pombe and $16 \mathrm{~L}$. kluyveri strains (see Materials and Methods). The $S$. pombe reference genome encodes two introns in cox 1 and a single intron in cob. Importantly, the group II cob intron alone is known to be mobile (Zimmer et al. 1987). In L. kluyveri, a recent study found evidence for mobility of both cox 1 and cob introns, noting presence/absence polymorphisms for three out of four $c o b$ and three out of five cox1 introns (Jung et al. 2012). In line with widespread mobility in this species, all introns with the exception of the first $c o b$ intron encode endonucleases (Friedrich et al. 2012). As predicted under a model where gene identity is secondary but mobility plays a pivotal role in nonrandom nucleotide diversity at intron-exon boundaries, we observe SNP density gradients across both $\operatorname{cob}(\tau=-0.21$, $P=0.03$, Figure 2D) and cox 1 exons $(\tau=-0.29, P=0.002)$ in L. kluyveri, whereas in $S$. pombe a negative SNP gradient is evident for $\operatorname{cob}(\tau=-0.31, P=0.002)$ but not $\operatorname{cox} 1$ ( $\tau=0.16, P=0.1$ ).

\section{No evidence for relaxed purifying selection at intron-exon boundaries}

A number of evolutionary scenarios might account for elevated exonic nucleotide diversity at sites of intron gain and loss. Importantly, mobility need not be causal: introns might instead be located in areas that are under reduced selective constraint. To investigate whether homing sites might be biased toward regions under lower functional constraint, we considered the ratio of nonsynonymous to synonymous changes as an indicator of protein-level selection. In L. kluyveri, within 20 nt of the intron-exon boundaries of cox 1 and $c o b$ only 6 out of 29 SNPs (21\%) are nonsynonymous, a significant depletion compared to the mutational expectation of $\sim 2 / 3$ (Fisher test $P=0.001$ ). Similarly, only one out of eight SNPs (12.5\%) in close vicinity of the $S$. pombe cob intron is nonsynonymous (Fisher test $P=0.12$, but note that power here is limited by the small number of mutations). In both cases, lower levels of nonsynonymous diversity support the notion of strong ongoing protein-level selection at intronexon boundaries. Interestingly, we find a relatively large 
number of nonsynonymous mutations in $S$. cerevisiae cox 1 $(15 / 44=34 \%)$. However, the ratio is similarly high $(5 / 19=$ $26 \%$ ) further away from the boundary (Fisher test $P=0.77$ ), arguing for a global rather than local, boundary-anchored relaxation of constraint. This observation is broadly consistent with prior evidence for reduced selection on mitochondria in the wake of the whole genome duplication (WGD) event (Jiang et al. 2008). We note that, in this regard, $S$. cerevisiae and other post-WGD species might be uniquely informative for assessing mutational forces at work in mitochondrial protein-coding genes.

Further testimony for ongoing purifying selection at the intron-exon boundary comes from scrutinizing exonic residues involved in base-pairing interactions with the neighboring intron (see Materials and Methods). Disruption of proper intron-exon base pairing is anticipated to impair splicing, with deleterious consequences for the host since splicing is required to reconstitute functional $c o b / c o x 1$ reading frames. Although splice-relevant exonic residues are firmly located in the zone of enriched SNP density, we find only a single SNP at a nucleotide position that is involved in intron-exon base pairing; and even this SNP, a synonymous change (CAC $\leftrightarrow$ CAT) at the 5 ' end of $\operatorname{cox} 1$ exon 5 , likely preserves base pairing (G-C $\leftrightarrow \mathrm{G}-\mathrm{T}$ ). This observation supports the notion that we do not see higher SNP densities as the result of relaxed selection but rather despite persistent functional constraint. In fact, if excess variation reflects differential mutational input, we are likely to underestimate the true mutational gradient, given the added splicing-related constraints in the immediate vicinity of introns.

Finally, to provide a further, complementary layer of evidence that variation in local conservation is not the cause of excess genetic diversity in the vicinity of mobile introns, we make use of the highly conserved nature of ETC proteins across eukaryotes and consider local topologies of constraint in human cox 1 and $c o b$ (also known as $c y t b$ ). We reasoned that local constraints on protein function and structure should be very similar between the human and yeast orthologs and that, in considering polymorphisms found in the constitutively intronless human orthologs, we circumvent potential circularity in assessing the relationship between introns and local conservation. We therefore charted SNP density across human cox 1 and $c o b$ as a function of distance from mock splice junctions, placed at orthologous positions in the respective gene (see Materials and Methods). We find no evidence for locally relaxed selective constraint for either gene, regardless of whether we assume $S$. cerevisiae or L. kluyveri intron positions (Figure 3). If anything, SNP densities are somewhat lower close to the insertion site, consistent with previous reports that self-splicing introns will be more successful if they integrate into functionally conserved sequence contexts since this prevents the host from escaping by mutating away from the recognition motif and opens up colonization opportunities in other species (Goddard and Burt 1999; Koufopanou et al. 2002). Overall, the evidence presented above is inconsistent with locally reduced selection and instead points to a causal contribution of mobility in generating observed diversity patterns.

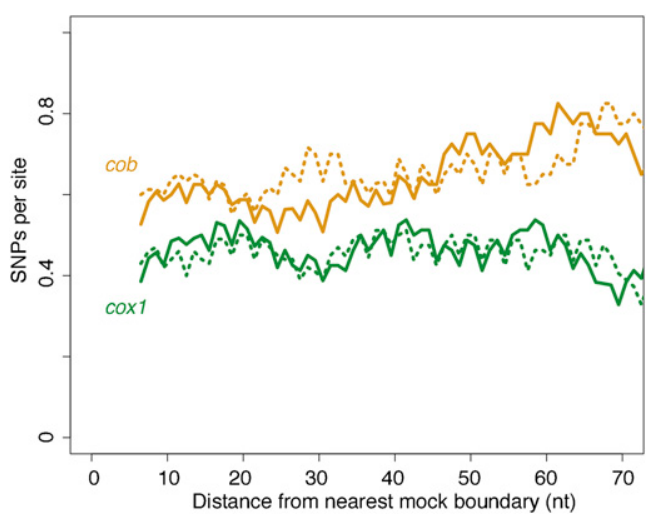

Figure 3 No evidence for locally relaxed purifying selection at intronexon boundaries. SNP density across human cob (yellow) and cox 1 (green) coding sequences as a function of mock intron-exon boundaries introduced in silico (see main text), based on either intron positions in S. cerevisiae (solid lines) or L. Kluyveri (dotted lines). Note that the 3-nt periodicity reflects the fact that the majority of introns occur in the same phase. The trend lines are smoothed density curves as described in Figure 1B.

\section{Candidate molecular mechanisms for mobility-associated SNP patterns}

Which mobility-associated molecular processes might lead to elevated SNP rates at intron-exon boundaries? One candidate mechanism is gene conversion. Previous studies in yeast (Zinn and Butow 1985), and notably also plants (Cho et al. 1998; Sanchez-Puerta et al. 2011), have provided experimental and comparative genomic support for exon coconversion. After endonuclease activity has introduced a DSB into the intron-free locus, exonucleases resect part of the neighboring exon and genetic information not previously present is introduced from the uncleaved repair template. The length of the resected fragment varies, with exonic portions closer to the intron more frequently affected (Mueller et al. 1996). However, more frequent conversion of intron-proximal exonic sequence does not by itself explain higher diversity in that region. This is because gene conversion only shuffles preexisting genetic diversity. If it occurs between genomes in the same recombining population, no additional variation is introduced that would account for a greater incidence of SNPs near intron-exon boundaries. Thus, for exon coconversion to explain our data, an important additional requirement needs to be met: novel variants must be introduced into the population from the outside. That is, the template for conversion needs to be introduced via horizontal gene transfer (HGT) or introgression events. And since we are considering diversity within extant populations, this process has to be ongoing (or at least recent) and pervasive (i.e., affecting several introns). Such a scenario is not necessarily unreasonable, given prior findings, notably in plants, of high rates of horizontal transfer of self-splicing introns (Cho et al. 1998; Goddard and Burt 1999; Strope et al. 2015). However, we were unable to identify likely donors for such putative HGT events, despite a comprehensive survey of NCBI's nonredundant nucleotide database. 
An alternative to the HGT plus exonic coconversion model is that intron insertion sites constitute mutational hotspots, as previously suggested (Hensgens et al. 1983; Zimmer et al. 1987; Foury et al. 1998). There are two major scenarios of how mutations might be generated as a side effect of intron mobility. In the first scenario, mutations are introduced when intron-free mRNAs or intron-containing pre-mRNAs are converted into cDNA by a resident error-prone reverse transcriptase, and that cDNA mediates precise intron loss or intron gain, respectively. This process can in principle act in trans and impact introns other than those specifically encoding ORFs with RT activity. However, importantly, reverse transcription does not predict a higher mutation load at intron-exon boundaries. In the second scenario, novel variants are produced by mutagenic repair following endonuclease-mediated cleavage. Interestingly, there is some prior evidence-based on studies of the $S$. cerevisiae MAT locusthat DSB repair in the context of endonuclease-mediated cleavage is mutagenic. Yu and Gabriel (2003) found deletions in the $\mathrm{Z}$ region, which borders the cutting site of the $\mathrm{HO}$ endonuclease, in a high proportion $(2 \%)$ of yeast crosses, which were attributed to microhomology-mediated end joining. Further, studying HR, Hicks et al. (2010) noted high (1400-fold over spontaneous) rates of predominantly single nucleotide mutations, which they attributed to the action of error-prone polymerases (Hicks et al. 2010). Given these prior observations, we examined polymorphisms in the Z1 region of the MAT locus but found it to be perfectly conserved across the $92 \mathrm{~S}$. cerevisiae strains analyzed here. The first SNP was found 192 nt downstream of the HO cutting site. This high level of conservation is indicative of exceptionally strong nucleotide-level constraint and echoes previous observations of very slow divergence ( $>96 \%$ nucleotide identity) between otherwise well-diverged Saccharomyces spp. (Gordon et al. 2011). Unfortunately, this precludes the use of natural diversity at the MAT locus as a model to study mutagenic effects.

It is worth considering at this point whether the finding that HO-initiated DSB repair is mutagenic might be specifically reflective of HR following endonuclease-mediated cleavage rather than HR in general. Is it possible that the activity or presence of the endonuclease itself affects the repair process? There have been a number of recent reports that DNA-binding proteins, by associating with a lesioncontaining target site, can prevent proper damage surveillance and repair, ultimately leading to a higher incidence of mutations (Reijns et al. 2015; Kaiser et al. 2016; Sabarinathan et al. 2016). Endonucleases, which bind their recognition motifs with high affinity, might elicit similar effects, for example by competing with the repair machinery when DSB repair is being templated by a second intron-free copy of the mitochondrial genome, which is also at risk of being cleaved. Indeed, there is some evidence of repair interference from the self-splicing $t d$ intron of phage T4, where the intron-encoded I-TevI endonuclease, which cleaves distally to its binding site, remains preferentially associated with one of the free cleavage products, and thereby asymmetrically impedes resection (Mueller et al. 1996). We posit that endonuclease activity remains an intriguing candidate for the mechanism behind mobilityassociated mutagenicity and warrants further investigation. We also suggest that, although HR is generally considered to be relatively error-free, HR in the context of endonucleasemediated cleavage might follow systematically different repair dynamics - a hypothesis that deserves additional experimental scrutiny, given the central role of targetable endonucleases in contemporary biotechnology.

If nonrandom patterns of genetic diversity are indeed mutational in origin, our findings have important implications for the cost of self-splicing introns, which have generally been considered as relatively cost-free, given their ability to efficiently remove themselves from their host genes (Werren 2011). Our results would suggest instead that these introns impose a mutational load on their host genes in addition to potential physiological costs, such as the energy and time expended in the splicing process. In as far as this mutational load affects fitness, the mutagenic effect might codetermine what constitutes evolutionarily sustainable insertion sites. That is, long-term safe havens for selfsplicing introns may be limited to regions within genes that exhibit sufficient functional constraint so that the host cannot mutate away from the cleavage site but are also robust enough to tolerate mutations introduced with some regularity by mutagenic activity. If, on the other hand, exonic coconversion is responsible for intron-proximal SNP gradients, our results strongly argue for ongoing cross-species transfer of mobile introns in extant yeast populations on a previously unrecognized scale.

\section{Acknowledgments}

We thank John Wolters, Paul Jung, Daniel Jeffares, Jonathan Flowers, Ashley Farlow, and Detlef Weigel for sharing sequence data and alignments, and Peter Sarkies, Anita Krisko, Daven Presgraves, and an anonymous reviewer for comments on the manuscript. T.W. is the recipient of intramural funding from the United Kingdom Medical Research Council and an Imperial College Junior Research Fellowship. The authors declare that they have no conflicts of interest.

Author contributions: J.R. and T.W. performed data analysis. T.W. conceived the study, designed analyses, and wrote the manuscript.

\section{Literature Cited}

Bonen, L., 2008 Cis- and trans-splicing of group II introns in plant mitochondria. Mitochondrion 8: 26-34.

Cao, J., K. Schneeberger, S. Ossowski, T. Günther, S. Bender et al., 2011 Whole-genome sequencing of multiple Arabidopsis thaliana populations. Nat. Genet. 43: 956-963.

Cho, S. W., S. Kim, Y. Kim, J. Kweon, H. S. Kim et al., 2014 Analysis of off-target effects of CRISPR/Cas-derived RNA-guided endonucleases and nickases. Genome Res. 24: 132-141. 
Cho, Y., Y. L. Qiu, P. Kuhlman, and J. D. Palmer, 1998 Explosive invasion of plant mitochondria by a group I intron. Proc. Natl. Acad. Sci. USA 95: 14244-14249.

Cohen, N. E., R. Shen, and L. Carmel, 2012 The role of reverse transcriptase in intron gain and loss mechanisms. Mol. Biol. Evol. 29: 179-186.

Cong, L., F. A. Ran, D. Cox, S. Lin, R. Barretto et al., 2013 Multiplex genome engineering using CRISPR/Cas systems. Science 339: 819-823.

Edgar, R. C., 2004 MUSCLE: multiple sequence alignment with high accuracy and high throughput. Nucleic Acids Res. 32: 1792-1797.

Foury, F., T. Roganti, N. Lecrenier, and B. Purnelle, 1998 The complete sequence of the mitochondrial genome of Saccharomyces cerevisiae. FEBS Lett. 440: 325-331.

Friedrich, A., P. P. Jung, J. Hou, C. Neuvéglise, and J. Schacherer, 2012 Comparative mitochondrial genomics within and among yeast species of the Lachancea genus. PLoS ONE 7: e47834.

Goddard, M. R., and A. Burt, 1999 Recurrent invasion and extinction of a selfish gene. Proc. Natl. Acad. Sci. USA 96: 13880-13885.

Gordon, J. L., D. Armisén, E. Proux-Wéra, S. S. ÓhÉigeartaigh, K. P. Byrne et al., 2011 Evolutionary erosion of yeast sex chromosomes by mating-type switching accidents. Proc. Natl. Acad. Sci. USA 108: 20024-20029.

Hensgens, L. A., L. Bonen, M. de Haan, G. van der Horst, and L. A. Grivell, 1983 Two intron sequences in yeast mitochondrial COX1 gene: homology among URF-containing introns and strain-dependent variation in flanking exons. Cell 32: 379-389.

Hicks, W. M., M. Kim, and J. E. Haber, 2010 Increased mutagenesis and unique mutation signature associated with mitotic gene conversion. Science 329: 82-85.

Jacquier, A., and B. Dujon, 1985 An intron-encoded protein is active in a gene conversion process that spreads an intron into a mitochondrial gene. Cell 41: 383-394.

Jeffares, D. C., C. Rallis, A. Rieux, D. Speed, M. Převorovský et al., 2015 The genomic and phenotypic diversity of Schizosaccharomyces pombe. Nat. Genet. 47: 235-241.

Jiang, H., W. Guan, D. Pinney, W. Wang, and Z. Gu, 2008 Relaxation of yeast mitochondrial functions after whole-genome duplication. Genome Res. 18: 1466-1471.

Jung, P. P., A. Friedrich, C. Reisser, J. Hou, and J. Schacherer, 2012 Mitochondrial genome evolution in a single protoploid yeast species. G3 2: 1103-1111.

Kaiser, V. B., M. S. Taylor, and C. A. Semple, 2016 Mutational biases drive elevated rates of substitution at regulatory sites across cancer types. PLoS Genet. 12: e1006207.

Kleinstiver, B. P., V. Pattanayak, M. S. Prew, S. Q. Tsai, N. T. Nguyen et al., 2016 High-fidelity CRISPR-Cas9 nucleases with no detectable genome-wide off-target effects. Nature 529: 490-495.

Koufopanou, V., M. R. Goddard, and A. Burt, 2002 Adaptation for horizontal transfer in a homing endonuclease. Mol. Biol. Evol. 19: $239-246$

Lambowitz, A. M., and M. Belfort, 1993 Introns as mobile genetic elements. Annu. Rev. Biochem. 62: 587-622.

Lazowska, J., B. Meunier, and C. Macadre, 1994 Homing of a group II intron in yeast mitochondrial DNA is accompanied by unidirectional co-conversion of upstream-located markers. EMBO J. 13: 4963-4972.

Levra-Juillet, E., A. Boulet, B. Séraphin, M. Simon, and G. Faye, 1989 Mitochondrial introns aI1 and/or aI2 are needed for the in vivo deletion of intervening sequences. Mol. Gen. Genet. 217: 168-171.

Lott, M. T., J. N. Leipzig, O. Derbeneva, H. M. Xie, D. Chalkia et al., 2013 mtDNA variation and analysis using mitomap and mitomaster. Curr. Protoc. Bioinformatics 44: 1.23.1-26.

Mali, P., L. Yang, K. M. Esvelt, J. Aach, M. Güell et al., 2013 RNAguided human genome engineering via Cas9. Science 339: 823-826.
Moran, J. V., C. M. Wernette, K. L. Mecklenburgh, R. A. Butow, and P. S. Perlman, 1992 Intron $5 \alpha$ of the COXI gene of yeast mitochondrial DNA is a mobile group I intron. Nucleic Acids Res. 20: 4069-4076.

Mueller, J. E., D. Smith, and M. Belfort, 1996 Exon coconversion biases accompanying intron homing: battle of the nucleases. Genes Dev. 10: 2158-2166.

Paschke, T., P. Meurer, and B. Schäfer, 1994 Homing of mitochondrial introns in the fission yeast Schizosaccharomyces pombe. Endocytobiosis Cell Res. 10: 205-213.

Reijns, M. A. M., H. Kemp, J. Ding, S. M. de Procé, A. P. Jackson et al., 2015 Lagging-strand replication shapes the mutational landscape of the genome. Nature 518: 502-506.

Roberts, R. J., T. Vincze, J. Posfai, and D. Macelis, 2010 REBASE: a database for DNA restriction and modification: enzymes, genes and genomes. Nucleic Acids Res. 38: D234-D236.

Rodgers, K., and M. McVey, 2016 Error-prone repair of DNA double-strand breaks. J. Cell. Physiol. 231: 15-24.

Sabarinathan, R., L. Mularoni, J. Deu-Pons, A. Gonzalez-Perez, and N. López-Bigas, 2016 Nucleotide excision repair is impaired by binding of transcription factors to DNA. Nature 532: 264-267.

Sanchez-Puerta, M. V., C. C. Abbona, S. Zhuo, E. J. Tepe, L. Bohs et al., 2011 Multiple recent horizontal transfers of the cox1 intron in Solanaceae and extended co-conversion of flanking exons. BMC Evol. Biol. 11: 277.

Sargueil, B., D. Hatat, A. Delahodde, and C. Jacq, 1990 In vivo and in vitro analyses of an intron-encoded DNA endonuclease from yeast mitochondria. Recognition site by site-directed mutagenesis. Nucleic Acids Res. 18: 5659-5665.

Strope, P. K., D. A. Skelly, S. G. Kozmin, G. Mahadevan, E. A. Stone et al., 2015 The 100-genomes strains, an S. cerevisiae resource that illuminates its natural phenotypic and genotypic variation and emergence as an opportunistic pathogen. Genome Res. 25: 762-774.

van Overbeek, M., D. Capurso, M. M. Carter, M. S. Thompson, E. Frias et al., 2016 DNA repair profiling reveals nonrandom outcomes at Cas9-mediated breaks. Mol. Cell 63: 633-646.

Wenzlau, J. M., R. J. Saldanha, R. A. Butow, and P. S. Perlman, 1989 A latent intron-encoded maturase is also an endonuclease needed for intron mobility. Cell 56: 421-430.

Wernette, C., R. Saldanha, D. Smith, D. Ming, P. S. Perlman et al., 1992 Complex recognition site for the group I intron-encoded endonuclease I-SceII. Mol. Cell. Biol. 12: 1903.

Werren, J. H., 2011 Selfish genetic elements, genetic conflict, and evolutionary innovation. Proc. Natl. Acad. Sci. USA 108(Suppl. 2): 10863-10870.

Wolters, J. F., K. Chiu, and H. L. Fiumera, 2015 Population structure of mitochondrial genomes in Saccharomyces cerevisiae. BMC Genomics 16: 281

Yu, X., and A. Gabriel, 2003 Ku-dependent and Ku-independent end-joining pathways lead to chromosomal rearrangements during double-strand break repair in Saccharomyces cerevisiae. Genetics 163: 843-856.

Zhou, Y., C. Lu, Q. J. Wu, Y. Wang, Z. T. Sun et al., 2007 GISSD: group I intron sequence and structure database. Nucleic Acids Res. 36: D31-D37.

Zimmer, M., F. Welser, G. Oraler, and K. Wolf, 1987 Distribution of mitochondrial introns in the species Schizosaccharomyces pombe and the origin of the group II intron in the gene encoding apocytochrome b. Curr. Genet. 12: 329-336.

Zinn, A. R., and R. A. Butow, 1985 Nonreciprocal exchange between alleles of the yeast mitochondrial 21S rRNA gene: kinetics and the involvement of a double-strand break. Cell 40: 887-895.

Communicating editor: D. C. Presgraves 


\section{GENETICS}

Supporting Information

http://www.genetics.org/content/early/2017/02/13/genetics.116.199059.supplemental

\section{Mobile Introns Shape the Genetic Diversity of Their Host Genes}

Jelena Repar and Tobias Warnecke 